\title{
Identification and Characterization of EX1 Kinetics in H/D Exchange Mass Spectrometry by Peak Width Analysis
}

\author{
David D. Weis, ${ }^{*}$ Thomas E. Wales, and John R. Engen \\ Department of Chemistry, University of New Mexico, Albuquerque, New Mexico, USA
}

\author{
Matthew Hotchko* and Lynn F. Ten Eyck \\ Department of Chemistry and Biochemistry and San Diego Supercomputer Center, University of California- \\ San Diego, La Jolla, California, USA
}

\begin{abstract}
Proteins that undergo cooperative unfolding events display EX1 kinetic signatures in hydrogen exchange mass spectra. The hallmark bimodal isotope pattern observed for EX1 kinetics is distinct from the binomial isotope pattern for uncorrelated exchange (EX2), the normal exchange regime for folded proteins. Detection and characterization of EX1 kinetics is simple when the cooperative unit is large enough that the isotopic envelopes in the bimodal pattern are resolved in the $\mathrm{m} / \mathrm{z}$ scale but become complicated in cases where the unit is small or there is a mixture of EX1 and EX2 kinetics. Here we describe a data interpretation method involving peak width analysis that makes characterization of EX1 kinetics simple and rapid. The theoretical basis for EX1 and EX2 isotopic signatures and the effects each have on peak width are described. Modeling of EX2 widening and analysis of empirical data for proteins and peptides containing purely EX2 kinetics showed that the amount of widening attributable to stochastic forward- and back exchange in a typical experiment is small and can be quantified. Proteins and peptides with both obvious and less obvious EX1 kinetics were analyzed with the peak width method. Such analyses provide the half-life for the cooperative unfolding event and the relative number of residues involved. Automated analysis of peak width was performed with custom Excel macros and the DEX software package. Peak width analysis is robust, capable of automation, and provides quick interpretation of the key information contained in EX1 kinetic events. (J Am Soc Mass Spectrom 2006, 17, 1498-1509) (C) 2006 American Society for Mass Spectrometry
\end{abstract}

$\mathrm{H}$ ydrogen exchange mass spectrometry (HXMS) can be used to distinguish different populations of molecules that exist during the exchange reaction [1-3]. The most obvious application of this ability is distinguishing the kinetic limits of exchange, or EX2 and EX1 kinetics. The majority of proteins are very stable under physiological conditions and, therefore, according to the models of HX $[4,5]$, must make many brief visits to a partially unfolded state to undergo exchange and become deuterated. Other proteins, perhaps with the help of denaturants or extremes of $\mathrm{pH}$, can undergo exchange via an EX1 mechanism wherein the exchange of a number of residues occurs simultaneously. The resulting mass spectra are quite distinct (Figure 1) as EX2 kinetics produces a

Published online July 27, 2006

Address reprint requests to Dr. J. R. Engen, Department of Chemistry, University of New Mexico, Clark Hall 242, MSC03-2060, Albuquerque, NM 87131-0001, USA. E-mail: engen@unm.edu

* These authors contributed equally to this work. gradual increase in average mass while EX1 kinetics results in the appearance of two distinct mass envelopes separated on the $m / z$ scale.

In biophysical terms, EX1 kinetics occur when a portion of the molecules being labeled undergo an unfolding event such that a number of residues are simultaneously exposed to deuterium in the labeling solution [4, 6-10]. Exchange of all the exposed residues occurs before the protein returns to the folded, less exchange-competent state. The rates of labeling, $k_{2}$, for the group of residues are greater than the refolding rate, $k_{-1}$, for the group (Figure 1). Such unfolding is termed cooperative or correlated exchange and is unusual for proteins under physiological conditions. As has been shown repeatedly (examples include: $[6,11-15]$ that the addition of denaturants or a change in $\mathrm{pH}$ (typically to a high value of 9.0-10.5) can artificially induce EX1 kinetics. While useful for understanding stability and the folding mechanism, artificially inducing EX1 kinetics generally does not reflect physiological protein dynamics. 

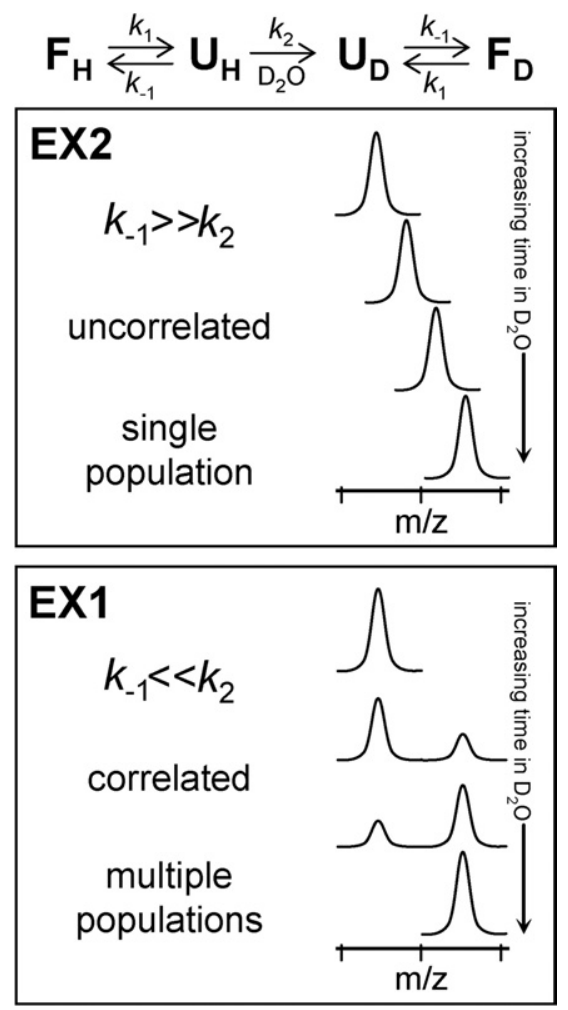

Figure 1. Protein unfolding and deuterium labeling in hydrogen exchange mass spectrometry $[1-8,31]$. The overall kinetic scheme is shown at the top and the resulting extremes of the two kinetic limits, EX2 and EX1, shown below.

A few proteins exhibit EX1 kinetics under physiological conditions. We have explored the EX1 phenomena in a collection of $\mathrm{SH} 3$ domains, some of which have obvious EX1 kinetics and others which do not [16]. While rare, the observation of physiological EX1 kinetics is of particular value, not only for understanding protein dynamics but also for the development of binding assays that exploit the unfolding and stability properties of the protein.

The appearance of EX1 kinetics in mass spectra is easy to diagnose for regions of proteins that have obvious separation between the folded/unlabeled population compared with the unfolded/labeled population (Figure 2a). However, it becomes increasingly difficult to characterize EX1 kinetics when the populations are less distinct (Figure $2 b-d$ ). Such a situation has been referred to as mixed-EX1 kinetics [2] or EXX kinetics [17]. In mixed kinetics, there may be EX2 and EX1 exchange occurring in a protein at the same time. Particularly when pepsin digestion is used, even long peptides may contain a portion that has undergone exchange via EX2 and a portion that has exchanged via EX1. If the number of residues involved in the EX1 unfolding is small, the distance between the two populations (Figure $2 \mathrm{~b}$ and $\mathrm{c}$ ) becomes small and processing becomes difficult. Most models assume that there are only two populations resulting from 2-state unfolding. However, there may be multiple populations as a result of multiple EX1 kinetic events, each with its own rates, occurring in different parts of large proteins.

As we illustrate in this work, EX1 kinetics can be characterized by measuring the peak width of the isotopic cluster. Such characterization is especially valuable for mixed kinetics, but can also be applied to EX1 signatures that are more resolved (Figure 2a). The use of several types of processing methods will be described and discussed including: fitting with Gaussians, measurement of isotope envelope width and automated EX1 identification, and width measurement with the DEX [18] software package.

\section{Methods \\ Materials and Deuterium Labeling}

Proteins were overexpressed, purified, and labeled as described previously $[16,19,20]$. Generally speaking, a stock solution of each protein at $100-200 \mathrm{pmol} / \mu \mathrm{L}$ in a physiological buffer (potassium phosphate, Tris, $\mathrm{pH}$ $7-8, \mathrm{H}_{2} \mathrm{O}$ ) was prepared. Deuterium exchange was initiated by dilution of the stock solution 15-fold with a $99 \%$ deuterium version of the same buffer at $\mathrm{pD} 7.0$, $\mathrm{D}_{2} \mathrm{O}, 21^{\circ} \mathrm{C}$. At each time point an aliquot from the exchange reaction was removed and labeling was quenched by adjusting the $\mathrm{pH}$ to 2.6 with an equal volume of quench buffer (such as $100 \mathrm{mM}$ potassium phosphate, $\mathrm{pH}$ 2.6, $\mathrm{H}_{2} \mathrm{O}$ ). Quenched samples were immediately frozen on dry ice and stored at $-80{ }^{\circ} \mathrm{C}$ until analysis.

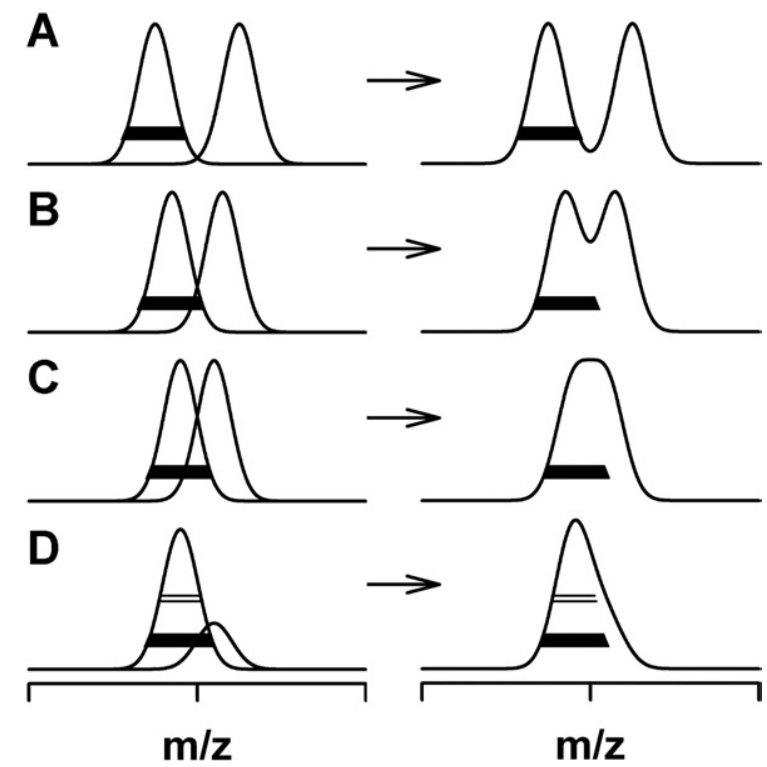

Figure 2. Merging of two Gaussian distributions of equal width but varying centers (in $\mathrm{m} / \mathrm{z}$ units). The separated distributions are shown on the left and the merged shown on the right. The solid bar is set at the width of a single Gaussian at $20 \%$ peak intensity while the open bar is set at $50 \%$ peak intensity. 


\section{Mass Analysis of Intact Proteins}

Labeled proteins were rapidly thawed at $0{ }^{\circ} \mathrm{C}, 200-300$ pmol of protein were injected onto a $\mathrm{C} 18$ protein trap and desalted for $3 \mathrm{~min}$ at $2 \% \mathrm{ACN}(0.05 \%$ TFA) at a flow rate of $200 \mu \mathrm{L} / \mathrm{min}$. The proteins were eluted into the mass spectrometer using a $2-98 \%$ ACN step in $0.2 \mathrm{~min}$ at $50 \mu \mathrm{L} / \mathrm{min}$ with a Shimadzu HPLC (LC-10ADvp, Columbia, MD). The HPLC was performed using protiated solvents which results in the removal of deuterium from the side chains and the amino/carboxy termini that exchange faster than backbone amide hydrogens [21]. All steps were performed with the C18 protein trap, injector and associated tubing submerged in an ice bath. Mass spectral analyses were carried out with a Waters QTOF2 mass spectrometer (Milford, MA) with a standard electrospray source. The deuterium levels were not corrected for back exchange $[8,21]$ and are therefore reported as relative. The average amount of back exchange for intact proteins using the experimental setup just described was $12-15 \%$ based on analysis of highly deuterated protein standards.

\section{Pepsin Digestion and Mass Analysis of Peptides}

Deuterium exchange was performed as described above. Before analysis, the quenched exchange sample was incubated with a 1:1 ratio (weight/weight) of protein:pepsin for $5 \mathrm{~min}$ on ice or digested with online pepsin columns as described [20,22]. The peptides were fractionated in $5 \mathrm{~min}$ by a $5-70 \% \mathrm{ACN}$-water gradient at a flow rate of $50 \mu \mathrm{L} / \mathrm{min}$ using a $50 \times 1.00 \mathrm{~mm}$ reversed-phase $\mathrm{C} 18$ column (such as Jupiter $4 \mu$ Proteo $90 \AA$, Phenomenex, Torrance, CA) maintained at $0{ }^{\circ} \mathrm{C}$. Analyses were performed with the same instrument as above. The deuterium levels were not corrected for back exchange $[8,21]$ and are therefore reported as relative. The average amount of back exchange for peptides using the experimental setup described was $18-20 \%$ based on analysis of highly deuterated peptide standards. All peptide mass assignments were confirmed by exact mass measurements and/or MS/MS experiments.

\section{Peak Width Determination}

For intact proteins $>10 \mathrm{kDa}$ analyzed by time of flight MS (QTOF2), the typical charge state envelope is an almost smooth, unresolved feature. Peak width at a particular percentage of maximum intensity (e.g., 20\%) was determined either by transforming the charge-state envelope and measuring the width of the resulting uncharged protein peak or by measuring the width of a particular charge state envelope. In the latter case, the width in $\mathrm{m} / \mathrm{z}$ units was then converted to a width in Daltons by multiplying by the charge state of the measured peak. Both types of measurements were done using the mass spectrometry processing software (MassLynx, Waters, Milford, MA). For peptide spectra, in which the individual isotopic peaks are fully re- solved, peak width determination was more complicated. An empirical peak-to-peak envelope was laid over the isotopic envelope. The width of the empirical envelope was measured at $20 \%$ of the maximum intensity using a linear interpolation between the peak-topeak points of the envelope. Peak width determination was implemented as a Visual Basic Macro in Microsoft Excel (available from the authors). The process is described in detail in the Appendix.

\section{Data Analysis with DEX}

The DEX package (http:/ / biology.sdsc.edu/ccms/dex) was used to deconvolute and analyze peptide data in an automated fashion, using a Fourier transform method to extract the backbone amide populations from the original mass spectrometry data [18]. Improvements to the DEX package since it was first described allowed for the analysis of electrospray mass spectrometry data containing multiply-charged ions. DEX automatically chose an interval necessary to maintain a sufficient number of points between isotopic peaks. For the data analyzed here, the number of points between isotopic peaks was 10 in every case. The DEX software automatically calculated centroid values as determined by the individual backbone amide population values for each peptide time point. In addition, a new peak width analysis method was developed by using a similar approach to the one described above. The main difference is the peak width method used by the DEX package first integrates each population peak before the linear interpolation is calculated. The peak width measurement can be made for both the deconvoluted backbone amide populations and the original mass spectrum.

\section{Modeling EX2 Exchange}

To estimate the magnitude of widening under EX2 conditions, a simple model of $\mathrm{H} / \mathrm{D}$ exchange was developed. In the model, polypeptides (peptides or proteins) were treated as having a combination of rapidly-, intermediately-, and non-exchanging amide hydrogens (NHs). Rapidly-exchanging NHs were fully exchanged during the forward exchange step and nonexchanging NHs did not undergo exchange. Intermediate exchange was treated as a first-order reaction with a single rate constant of $0.0693 \mathrm{~min}^{-1}\left(t_{1 / 2}=10 \mathrm{~min}\right)$. The probability of forward exchange, $p_{\mathrm{fwd}}$, as a function of time, $t$, is then simply

$$
p_{\mathrm{fwd}}=1-e^{-k t}
$$

Back-exchange probability, $p_{\text {back }}$, was treated as uniform for all exchanged amides and fixed at 18\% (based on the values typically observed experimentally).

For simplicity, in the following treatment, the mass of the polypeptide is considered in terms of offsets above the monoisotopic mass [18]. Since both forward 
and back exchange are stochastic processes under the EX2 kinetic mechanism, the forward and back exchange produce a distribution of deuteration states described by the binomial distribution function $P$

$$
P(n, N, p)=\frac{N !}{n !(N-n) !} p^{n}(1-p)^{N-n}
$$

where $n$ is the number of either forward exchanged NHs $\left(n_{\text {fwd }}\right)$ or back-exchanged NDs $\left(n_{\text {back }}\right), N$ is the total possible number of exchange events, and $p$ is the probability of either forward or back exchange.

For forward exchange, $N$ is equal to the number of $\mathrm{NHs}$ that exchange on the intermediate timescale, $N_{\text {int }}$. For back exchange, $N$ is equal to the sum of the rapidly exchanged NHs, $N_{\text {fast}}$ and the number of $\mathrm{NHs}$ that exchange on the intermediate timescale, $N_{\text {int }}$. The final level of deuteration is then simply

$$
n=n_{\mathrm{fwd}}-n_{\text {back }}
$$

Application of the binomial distribution to forward exchange, $F(n)$, is trivial,

$$
F(n)=P\left(n, N_{\text {int }}, p_{\text {fwd }}\right)
$$

but inclusion of back exchange requires a convolution of forward and back exchange, since more than one forward exchange/back exchange scenario can yield the same final deuteration level. For example, $\left(n_{\mathrm{fwd}}=5\right.$, $\left.n_{\text {back }}=2\right)$ and $\left(n_{\text {fwd }}=3, n_{\text {back }}=0\right)$ both produce the same final deuteration of $n=3$, though the probabilities of these two scenarios are different. Convolution is achieved by considering all possible combinations of $n_{\mathrm{fwd}}$ and $n_{\mathrm{back}}$ that produce a given level of deuteration $n, B(n)$ :

$$
B(n)=\sum_{i=n}^{N_{\max }} P\left(i-N_{\text {fast }}, N_{\text {int }}, p_{\text {fwd }}\right) \times P\left(i-n, i, p_{\text {back }}\right)
$$

where $N_{\max }=N_{\text {int }}+N_{\text {fast }}+N^{\prime}$, and $N^{\prime}$ is the offset between the monoisotopic mass and the largest mass considered in the natural isotopic distribution (see below) [18]. Unphysical exchange situations (e.g., $n_{\text {back }}$ $>n_{\mathrm{fwd}}$ or $\left.n_{\mathrm{fwd}}>N_{\mathrm{int}}\right)$ are excluded by defining $P(n, N$, $p)=0$ for $n>N$ and for $n<0$.

A similar method was used to convolute the natural isotopic distribution, $I(n)$, with $B(n)$. Here, the natural isotopic distribution is treated as a series of mass offsets above the monoisotopic mass. For example, incorporation of one ${ }^{13} \mathrm{C}$ results in a +1 offset. As with back exchange, more than one combination gives rise to the same observed mass. For example, a final deuteration level of 5 for the monoisotopic peak gives the same observed mass as a deuteration level of 4 on the +1 isotopic peak. Natural isotopic distributions were calculated using either the DEX software package [18] or MassLynx (Waters). The final observed population of deuteration level $n, D(n)$, is

$$
D(n)=\sum_{i=0}^{n} B(i) \times I(n-i)
$$

where $\mathrm{I}(n)=0$ for $n>\mathrm{N}^{\prime}$.

For some calculations, a more complex EX2 exchange model in which the amides that exchanged on the intermediate time scale were subdivided into three populations $\left(N_{\text {int, } 1}, N_{\text {int,2, }}\right.$ and $\left.N_{\text {int, } 3}\right)$ that exchanged at three different rates determined by the first-order rate constants $k_{1}=0.693 \mathrm{~min}^{-1}, k_{2}=0.0693 \mathrm{~min}^{-1}$, and $k_{3}=$ $0.00693 \mathrm{~min}^{-1}$. In this case, the forward exchange population distribution $F(n)$ is obtained by a convolution of the three exchanging populations:

$$
\begin{aligned}
& F(n)=\sum_{i=0}^{n} \sum_{j=0}^{n-i} P\left(i, N_{\mathrm{int}, 1}, p_{x}\right) \times P\left(j, N_{\mathrm{int}, 2,}, p_{y}\right) \\
& \times P\left(n-i-j, N_{\mathrm{int}, 3,}, p_{z}\right)
\end{aligned}
$$

where $p_{1}, p_{2}$, and $p_{3}$ are obtained from eq 1 with $k_{1}, k_{2}$, and $k_{3}$, respectively. Following convolution, the masses were offset by $N_{\text {fast }}$ :

$$
F^{\prime}(n)=F\left(n-N_{\text {fast }}\right)
$$

where $F^{\prime}(n)=0$ for $n<N_{\text {fast }}$. The forward exchange population, $F^{\prime}(n)$, was then convoluted with back exchange and the natural isotopic abundance as described previously.

These exchange models were implemented as Visual Basic for applications macros (available from the authors) running in Microsoft Excel (Redmond, WA).

\section{Results and Discussion}

Uncovering EX1 kinetics in proteins at physiological conditions provides information about native protein dynamics. Once observed, the properties that become valuable are the rate of the unfolding event and the number of residues involved. The location of the unfolding also becomes important and can often, but not always, be determined by analysis of peptic fragments produced after the exchange reaction is quenched. In many cases, a qualitative analysis can be performed first and the approximate rate and location defined. When one is interested in comparing multiple states of proteins [8] rather than determining the exact number of residues exchanging at particular rates for a single protein in isolation, no further characterization is required. A comparison of the effects of changing the proteins conditions (bound versus unbound, active versus inactive, etc.) will demonstrate the effects on EX1 kinetics, if any. If the exact number of residues involved is then later required, back-exchange correction can be performed and the results analyzed for the fragments of interest. This reverse methodology can save a tremendous amount of time by not requiring analysis of every fragment if most of them do not experience changes, although automated software approaches (discussed below) make this less of an issue. 


\section{Gaussian Distributions and EX1}

In a simple 2-state unfolding reaction displaying EX1 kinetics in mass spectra (Figure 2a), the most straightforward approach to determine the unfolding rate and the number of residues participating in the unfolding is by fitting Gaussian distributions to the isotope envelopes of the two populations (recently illustrated by [23]. By monitoring the decay of the natural log of the fraction of folded molecules (lower mass distribution) with time (first-order kinetics), the rate constant for the unfolding can be determined [24, 25]. While Gaussian modeling of EX1 is easy when the two populations are distinct, it becomes much more challenging when the two populations start to merge (Figure $2 b$ and $c$ ) and when the relative intensity or contribution to the overall population of one of the species is significantly less than the other (Figure 2d). The width of each Gaussian is often set at the width of the isotopic distribution before and/or after the EX1 signature, and then the intensity of the distributions and their centers are modified to fit the Gaussians to the experimental data (e.g., reference [23].). Multiple solutions can be obtained in this fitting process, depending on the quality of the original data. We believe that an analysis of the peak width over time provides much of the same information as Gaussian fitting and that it can be done with high confidence even for those types of distributions that show mixed kinetics (Figure $2 \mathrm{~b}-\mathrm{d}$ ). An added value of the peak width method versus Gaussian modeling is that peak width determinations make no assumptions. Gaussian modeling assumes that a 2-state model is followed, while the peak width method assumes nothing but rather simply places the raw data in a form that allows easy interpretation (examples below). Particularly in cases when one is interested in what happens when EX1 disappears, the no-assumption method is much faster and provides the same ultimate information.

\section{Does EX2 Kinetics Result in Peak Widening?}

One important consideration with peak width analysis is the contribution to the width of the isotopic cluster from the uptake of deuterium itself, regardless of the kinetic mechanism. It has been argued $[17,26]$ that one cannot use the relative change in peak width to diagnose the presence of EX1 kinetics because there is a stochastic widening (sometimes referred to as statistical widening) of peaks in EX2 exchange. Near the EX2 kinetic limit, exchange at any particular amide on any given molecule is a random event that is independent (uncorrelated) with exchange at any other location on any other molecule. Such a situation is described by a binomial distribution, which predicts that peptides or proteins will have a distribution of different deuterium levels. The reader is referred to a lucid treatment of this phenomenon by Arrington et al. [3]. Binomial statistics predict that the standard deviation of the distribution, $\sigma$, (i.e., the half-width at $e^{-1 / 2}$ of the maximum) is

$$
\sigma=\sqrt{n p(1-p)}
$$

where $n$ is the number of exchanging amide hydrogens, and $p$ is the probability of exchange. Differentiation with respect to $p$ shows that the width of the distribution will be widest at $p=0.5$, that is, at the midpoint of the exchange process and that it decreases on both sides of the midpoint. This, then, is the basis for the argument that stochastic widening is evident under EX2 exchange conditions. This type of widening is illustrated in more detail below.

Two methods (modeling and empirical measurements) will be used to illustrate that the effects of EX2 peak broadening are limited to a very small change in peak width. In the first approach (Figure 3a-d), models of EX2 exchange were applied to peptides and proteins to determine the theoretical width of the isotopic envelope after a set amount of forward exchange and back exchange had occurred. The consideration of back exchange [21] in these models is important as it is also a stochastic widening process that contributes significantly to the observed width.

Figure 3a shows the width changes (at 20\% peak intensity) for a hypothetical peptide with 15 exchangeable amide hydrogens that has the isotopic distribution of the peptide of sequence LAEANVMKTLQHDKL undergoing modeled EX2 exchange with various amounts of rapid-, intermediate-, and nonexchanging NHs (deuterium uptake curves for these model peptides are provided in the Supplementary Materials section which can be found in the electronic version of this article). Since the model assumes uniform exchange, the sequence is only needed so that a natural isotopic distribution can be determined. In the ensuing discussion, we will resort to a simplified notation to describe the number of amides that fall into the different exchange categories (i.e., rapid, intermediate, and non-exchanging) of the form $N_{\text {fast }} / N_{\text {int }} / N_{\text {non }}$ where $N_{\text {non }}$ represents amides that do not exchange. In the first exchange scenario, $0 / 15 / 0$, all 15 amides exchange with the same rate $(k$ $\left.=0.0693 \mathrm{~min}^{-1}, t_{1 / 2}=10 \mathrm{~min}\right)$. We begin with a treatment of EX2 exchange in which only the forward exchange process is considered (Figure 3a, open triangles). Here, there is a clear widening of the distribution with a sharp width maximum at the midpoint of the exchange process $\left(p=0.5, t_{1 / 2}=10\right.$ min). This represents stochastic widening of the distribution. When back exchange is taken into account (Figure 3a, filled diamonds), a sharp increase in width up to the midpoint is evident, but beyond the midpoint, the width tapers off more gently. At these longer exchange times, most of the amides undergo a significant amount of back exchange (because more amides have the possibility of being deuterated) which smoothes out the stochastic effect. Increasing back exchange levels (e.g., to 50\%) in the model calculations results in nearly constant peak width 
MODEL EX2

EMPIRICAL EX2

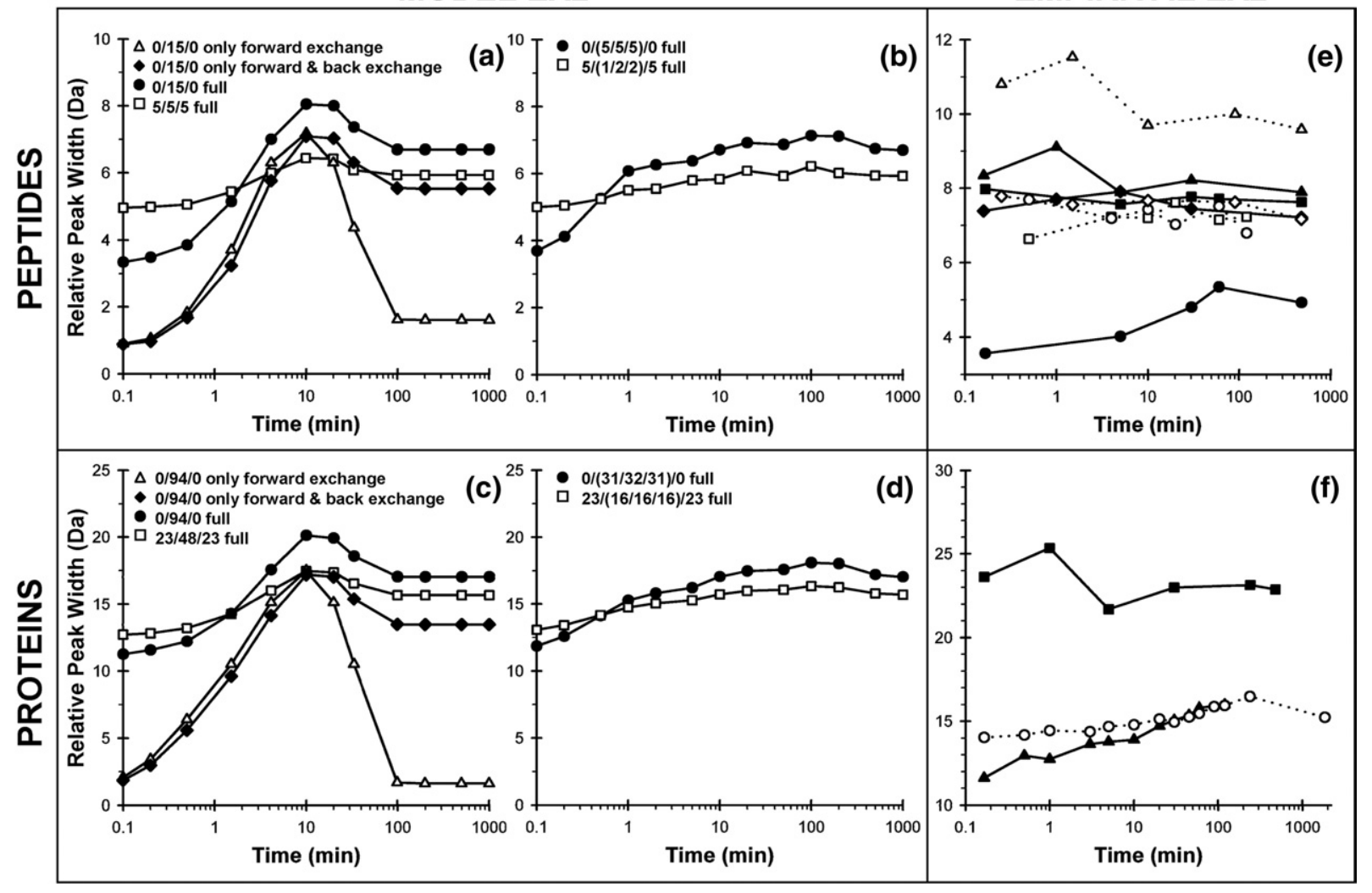

Figure 3. Changes in peak width at $20 \%$ of peak maximum for isotopic distributions displaying EX2 kinetics. Theoretical width changes determined from model calculations are shown in panels (a)-(d) and empirically determined width changes measured from a variety of peptides and proteins exhibiting EX2 kinetics are shown in (e-(f). In (a) and (c), the number of fast, intermediate and nonexchanging amide hydrogens are designated in by the notation $N_{\text {fast }} / N_{\text {int }} / N_{\text {non }}$. The width changes were calculated taking different aspects of the exchange process into account: only forward exchange, forward and back exchange, or a full calculation involving forward and back exchange and the isotopic distribution. In (b) and (d) the EX2 model uses three different exchange rates for the intermediate exchangers (see text for details) and the legend is therefore $N_{\text {fast }} /\left(N_{\text {int, } 1} / N_{\text {int }, 2} / N_{\text {int }, 3}\right) /$ $N_{\text {non }}$. Panel (e) shows empirically determined changes in width for eight peptides that display isotope patterns characteristic of EX2 kinetics. The data come from four unique proteins: (symbol, ion $\mathrm{m} / \mathrm{z}$, charge state, protein) filled square, 684, +3, DHFR; filled circle, 833, +1, DHFR; filled triangle, $890,+3$, HIV Nef; filled diamond, 1120, +2, HIV Nef; open square, 601, +3, HckYEEI; open circle, 855, +2, HckYEEI; open triangle, 1138, +3, Lck kinase domain; open diamond, 812, +2, Lck kinase domain. The width was determined at $20 \%$ relative intensity for all peptides. Panel (f) shows empirically determined changes in width for three unique proteins that display isotope patterns characteristic of EX2 kinetics. Filled triangle, Fyn SH3 domain [16]; open circle, Yes SH3 domain [16]; filled square, HIV Nef. The width of the transformed species was determined at $20 \%$ relative intensity for Fyn and Yes $\mathrm{SH} 3$ and at $65 \%$ relative intensity for HIV Nef (see text for details). The deuterium uptake curves for the peptides and proteins in panels (a)-(f) are available in the Supplementary Material Figure 1s. The raw mass spectra for the data in panels (e) and (f) are available in the Supplementary Material 2s-11s.

beyond the exchange midpoint (data not shown). Variations in back exchange due to peptide sequence and experimental conditions are unlikely to alter width changes that result from EX1 kinetic mechanisms. Taking the natural isotopic distribution into account (Figure 3a, filled circles) increases peak width primarily at early exchange times. Early in the exchange process, there is little stochastic widening and, thus, the natural isotopic abundance is the dominant contributor to the overall peak width. Finally, applying a more realistic $5 / 5 / 5$ scenario, in which five amides undergo rapid exchange, five exchange on an intermediate time scale, and five do not undergo exchange (Figure $3 a$, open squares), further suppresses the widening. Here, the fast exchangers do not contribute to widening during forward exchange, but do make a significant contribution during back exchange. Widening is further suppressed since only five amides exchange on the intermediate time scale. Even in this scenario though, there is a clear width maximum at $10 \mathrm{~min}$ with a gradual decrease on both sides.

While the modeling of EX2 kinetics just shown predicts some modest widening with a distinct maxi- 
mum at $50 \%$ exchanged, this model is a gross oversimplification of hydrogen exchange. First, it is unrealistic to simply divide the amide hydrogens into three bins (fast, medium, and non-exchanging). Second, even within the intermediate exchange regime, it is unreasonable to treat all $\mathrm{NHs}$ with a single first-order rate constant. Finally, it is well known that backbone amides exchange with different rates during back exchange [27-29]. All of these factors lead to a heterogeneous exchange process. This heterogeneity will attenuate the effects of stochastic widening. While a model of EX2 exchange taking all of these factors into account would be computationally demanding, a simple model is sufficient to justify this assertion. In Figure $3 b$, three rates of intermediate exchange are considered $\left(k_{1}=0.693\right.$ $\min ^{-1}, t_{1 / 2}=1 \mathrm{~min} ; k_{2}=0.0693 \mathrm{~min}^{-1}, t_{1 / 2}=10 \mathrm{~min}$; and $k_{3}=0.00693 \mathrm{~min}^{-1}, t_{1 / 2}=100 \mathrm{~min}$ ) for the same peptides as in Figure 3a. Peak width is nearly flat over the entire time course. In the case of 15 intermediate exchangers with $N_{\text {int }, 1}=5, N_{\text {int }, 2}=5$, and $N_{\text {int }, 3}=5$, (symbolized as $0 /(5 / 5 / 5) / 0$, filled circles), the maximum peak width increase is $\sim 3 \mathrm{Da}$, and the peak width varies by only $\sim 1 \mathrm{Da}$ over the 1 to $1000 \mathrm{~min}$ time scale. (In the exchange notation, parentheses group the intermediate rate exchangers.) When only five amides exchange over the intermediate time scale with $N_{\text {int }, 1}=1$, $N_{\text {int }, 2}=2$, and $N_{\text {int }, 3}=2$ (symbolized as $5 /(1 / 2 / 2) / 5$, open squares), the peak width change is $\sim 1$ Da over the entire time course. Equally important, there is no longer a sharp maximum. Thus, in a realistic model of EX2 exchange there is little evidence of peak width increase. The predicted stochastic widening (Figure 3a, open triangles) is suppressed because of the natural isotopic abundance (most important in the earliest time points), back exchange (most important at the latest time points), and amides that exchange at different rates (most important in the middle of the exchange process).

To model protein EX2 exchange, a hypothetical protein containing five of each of the 20 natural amino acids was used. Subtracting the five proline residues and the $\mathrm{N}$-terminus, there are a total of 94 exchangeable amides. Two models of EX2 exchange were investigated (Figure $3 \mathrm{c}$ and d), one with an extreme case of $94 \mathrm{NHs}$ exchanging during the intermediate timescale and the other more realistic one in which $23 \mathrm{NHs}$ exchange fast, $48 \mathrm{NHs}$ exchange at an intermediate rate, and 23 do not exchange at all (deuterium uptake curves for these model proteins are provided in the Supplementary Materials section). Figure $3 \mathrm{c}$ reprises, for proteins, the series of calculations shown in Figure 3a for peptides, in which all intermediate rate amides exchange with a single rate constant $\left(k=0.0693 \mathrm{~min}^{-1}, t_{1 / 2}=10 \mathrm{~min}\right)$. Figures $3 c$ reveals trends that are identical to those observed for the peptide calculations shown in Figures $3 a$, namely that widening is most pronounced when only forward exchange is considered and that there is only limited widening for a realistic number of exchangeable amides when back exchange and the natural isotopic abundance are considered. Figure $3 \mathrm{~d}$ shows how the peak width changes under model EX2 exchange conditions with three rate constants for intermediate exchange $\left(k_{1}=0.693 \mathrm{~min}^{-1}, t_{1 / 2}=1 \mathrm{~min} ; k_{2}=\right.$ $0.0693 \mathrm{~min}^{-1}, t_{1 / 2}=10 \mathrm{~min}$; and $k_{3}=0.00,693 \mathrm{~min}^{-1}$, $\left.t_{1 / 2}=100 \mathrm{~min}\right)$, similar to the peptide calculations shown in Figure 3b. Here, two scenarios are considered, one in which all 94 amides exchange in the intermediate exchange regime $\left(N_{\mathrm{int}, 1}=31 / N_{\mathrm{int}, 2}=32 / N_{\mathrm{int}, 3}=31\right)$ and a more realistic case with 48 amides exchanging on intermediate timescales $\left(N_{\text {int, } 1}=16 / N_{\text {int }, 2}=16 / N_{\text {int }, 3}=\right.$ 16). As is apparent from Figure $3 d$, the peak width increases by $\sim 5 \mathrm{Da}$ for the $0 /(31 / 32 / 31) / 0$ case (filled circles), with the increase limited to $\sim 2$ Da over the 1 to $1000 \mathrm{~min}$ time interval. In the more realistic $23 /(16 / 16 /$ 16) $/ 23$ case (open squares), with a total 48 intermediate exchangers, the peak width increase is limited to $\sim 2 \mathrm{Da}$ over the entire time interval.

Although the three intermediate exchange rate model is still an oversimplification, an important trend emerges from the analysis. That is, stochastic widening is greatly diminished under complex exchange conditions. It is reasonable that in the limit of $n$ exchange rates for $n$ amides, that peak widening might be completely abolished. Such an analysis for a full protein would be computationally demanding. However, such a calculation, without consideration of back exchange, has been carried out for the 13 slowest exchanging amides in the third domain of turkey ovomucoid (OMTKY3) using NMR-derived amide exchange rates for each amide [3]. Although the width change was not quantified, inspection of the simulated spectra shows no evidence of a peak width increase over the exchange time course.

Also of note is that the absolute maximum peak width is obtained from a single rate model and that this model also produces the largest change in peak width. Thus, a homogeneous model may serve as an easily calculated upper bound for stochastic peak widening.

\section{Empirical Measurements of Width Changes in EX2}

Because modeling of the contribution of width changes to isotopic envelopes draws on a number of assumptions about the exchange, we have measured the empirical width changes for a number of peptides and proteins to verify the models and to obtain more real-world estimates of the EX2 widening of peptide and protein peaks in HXMS analyses. Figure 3e displays the results for the measurement of the peak width changes during deuterium labeling time courses for eight peptic peptides taken from four different proteins that show characteristic EX2 kinetic signatures only (the raw mass spectra and deuterium uptake curves for these peptides are provided in Supplementary Materials section). The magnitude of the width is dictated by the mass of the peptide. Those of smaller mass are narrower than those with larger mass, as dictated by the 
increasing width of the natural isotopic abundance with increasing mass. From these empirical data, clearly the change in peak width for peptides undergoing EX2 exchange is somewhere between 0.5-2 $\mathrm{Da}$ and that there is no obvious maximum or trend in the experimental data.

Empirical width changes during EX2 exchange for proteins were also obtained (Figure 3f) for three different proteins (the raw spectra and deuterium uptake curves are provided in the Supplementary Materials). Peak width was measured at $20 \%$ relative intensity in the case of signals with little contribution from cation adducts or at $65 \%$ relative intensity in the case of noisy signals with overlap from cation adducts. The changes in peak width in proteins undergoing EX2 exchange is at worst $4 \mathrm{Da}$ and generally between 2-3 Da. As can be seen by the comparison between the theoretical models and the empirical results, the stochastic widening that occurs during forward and back exchange for both peptides and proteins undergoing exclusively EX2 kinetics does not account for significant changes in peak width. For the worst cases, peak-width widening is limited to about $2 \mathrm{Da}$ in peptides and $4 \mathrm{Da}$ in proteins.

\section{Peak Width Increases Due to EX1 Exchange Kinetics}

Increases in peak width can be attributed to EX1 kinetics and the appearance of multiple populations. As already illustrated in Figure 2, if mixed kinetics occur, the width of the distribution increases, even when two distinct populations can not be observed. Two cases will be used to illustrate how the measurement of peak width is informative: bimodal patterns in which there are two obvious contributing populations (obvious EX1, Figure 2b) and those in which the bimodal patterns are less obvious (Figure $2 \mathrm{c}$ and $\mathrm{d}$ ). The processing is illustrated in Figure 4 for an obvious EX1 case. The Lyn SH3 domain shown here undergoes partial unfolding and displays obvious EX1 kinetics with an unfolding halflife of about $17 \mathrm{~min}$ [16]. Figure 4a shows representative spectra (all raw mass spectra are available in Supplementary Material Figure 12s). The bimodal EX1 pattern is visually obvious. Once the average amount of deuterium has been determined for each exchange time point, the value is plotted versus time to yield the typical deuterium uptake curve (Figure $4 b$ ). The deuterium uptake has some sigmoidal character, indicative of a large increase in deuterium level during the unfolding event. In the process of determining the average deuterium level at each time point, one takes the centroid of the isotopic distribution for each isotopic envelope. When a peak becomes wider, one would normally take the entire width of the wider distribution and determine the centroid of the entire wider distribution. This centroiding process is complicated when it is not readily obvious where the peak begins and ends [such as when there is a small contribution from an emerging
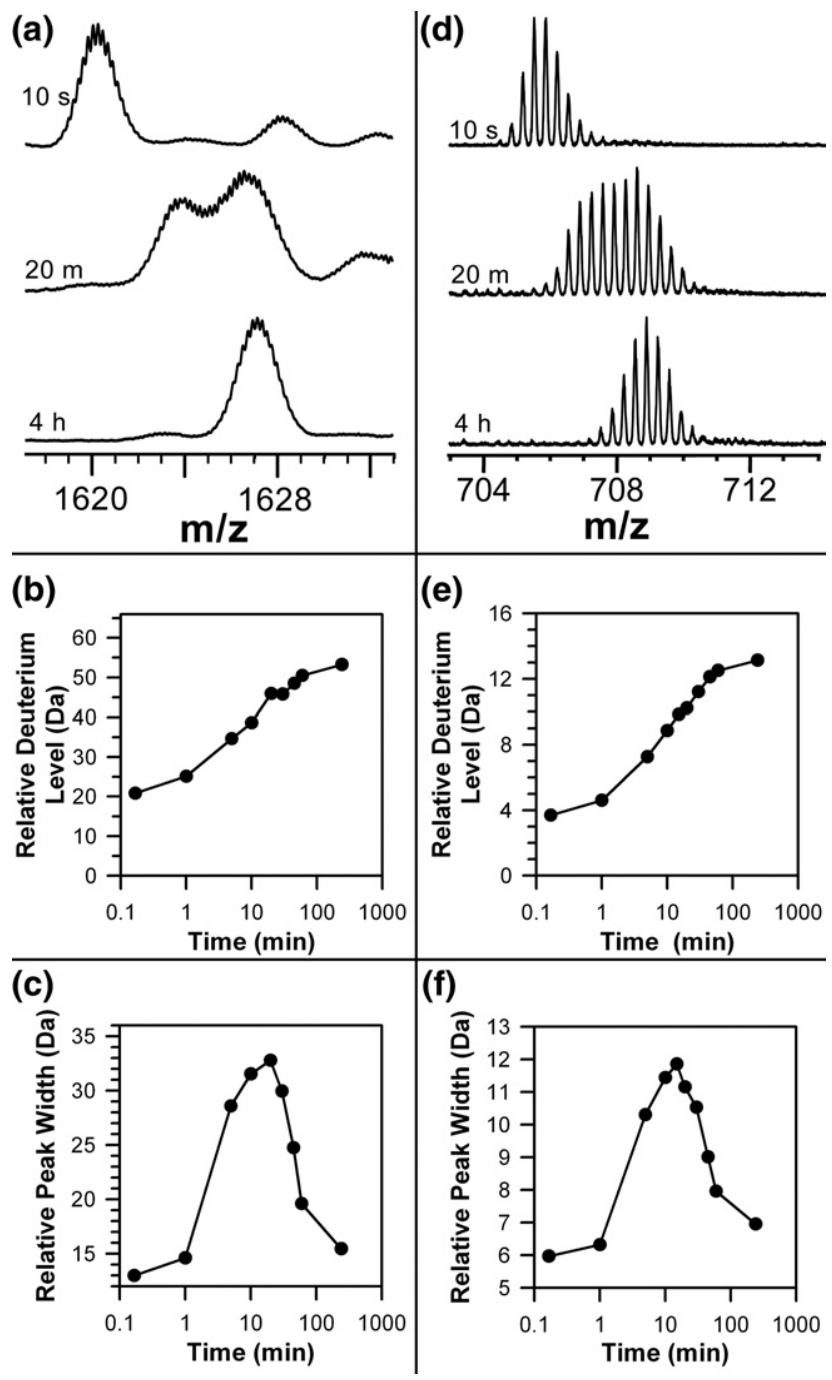

Figure 4. Width analysis of spectra displaying obvious EX1 kinetics. (a-c) Data for the Lyn SH3 protein [16] where (a) raw spectra for selected time points, (b) deuterium uptake curve, and (c) peak-width analysis. (d-f) Data for the Lyn SH3 peptide 99-116 [16] where (d) raw spectra for selected time points, (e) deuterium uptake curve, and (f) peak-width analysis. Peak width was determined at $20 \%$ intensity. All spectra for the entire time course for both protein and peptide are available in the Supplementary Material Figures 12s, 13s.

EX1 envelope (as in Figure 2d)]. If one plots the change in the width with time it becomes obvious that there has been a greater change to the width of the isotopic distribution with time than an exclusively EX2 model predicts. There are narrow peaks early on in the time course, the peak widens at some intermediate time value, and returns to a narrow form at later time points. The peak width analysis of the Lyn SH3 deuteration data is shown in Figure 4c. The graph is certainly not nearly as flat as was the case in Figure $3 \mathrm{f}$ for a protein with an exclusively EX2 kinetic signature. The change in peak width for Lyn SH3 is $\sim 20 \mathrm{Da}$, with the width returning to nearly the same place as it was before the unfolding event. The apex on the $y$-axis of such a width graph gives several pieces of information. The time 
value ( $x$-axis) when the apex occurs indicates the $t_{1 / 2}$ of the EX1 unfolding event. The change in the height of the peak from baseline values to the apex provides an estimate of the relative number of residues (or $\mathrm{m} / \mathrm{z}$ equivalent if not corrected for the charge state) in the unfolding unit within the protein that are involved in the EX1 kinetic event. In the examples shown here, these values are all considered relative because the deuterium level is relative (Figure 4b) as no back-exchange correction was made for these data (see Materials and Methods). If one corrected for back exchange and also had sufficient separation between the two envelopes (as in Figure 2a), the distance between the centroids of the two Gaussians would be equal to the number of residues that participated in the unfolding event. In peak width analysis, the distance between the centroids of the two hypothetical Gaussians is not explicitly determined so although the width change is proportional to the number of residues that participated, the exact number cannot be determined. Relative values, however, still provide valuable estimates.

As has been shown $[19,25,30]$, binding to a protein that shows EX1 kinetics can alter the unfolding kinetics and change the peak-width graph. Changes include shifts in the time the apex occurs and the magnitude of the rise in the $y$-axis direction. If peak widening resulted from the stochastic incorporation of deuterium, which we have shown in Figure 3 to be unrealistic, then peptide binding would not affect the peak-width graph (although binding may affect the deuterium uptake). The fact the binding alters the peak-width graph indicates that the biophysical basis for the shape of the plot is protein dynamics (in this case EX1 unfolding) that is altered by binding, not stochastic widening due to deuterium incorporation.

The analysis of the width changes in peptic peptides (Figure $4 \mathrm{~d}-\mathrm{f}$ ) follows a similar approach to that of the analysis of proteins (Figure 4a-c). The data shown in Figure 4 are for a peptic peptide (residues 99-116) from the Lyn SH3 domain, a peptide that participates in the unfolding illustrated for the whole protein. The half-life for unfolding in the peptide and protein is the same, as determined from the apex of the peak-width plot $(\sim 15$ mins). In obvious cases, such as that illustrated in Figure 4, the peak width change of over $6 \mathrm{Da}$ (Figure 4f) is well above the level of what is expected from stochastic widening (Figure 3a, b, e).

Peak width analysis is faster and simpler in almost all respects to the classical Gaussian fitting approach, although for obvious EX1 kinetics, the unfolding half-life and relative residue involvement can be obtained by Gaussian fitting (as in Figure 2b). Peak width analysis is particularly helpful and just as robust for cases where EX1 kinetics are less obvious (Figure 5). Again, we illustrate the analysis for a protein (Figure $5 \mathrm{a}-\mathrm{c}$ ) and for a peptide of that protein (Figure $5 \mathrm{~d}-\mathrm{f}$ ). Changes to the peak width are apparent (panels c and f) and well above the stochastic levels previously defined (Figure 3). One can quickly obtain the relative residue involvement and the half-life of unfolding simply by looking at the peak width graphs.
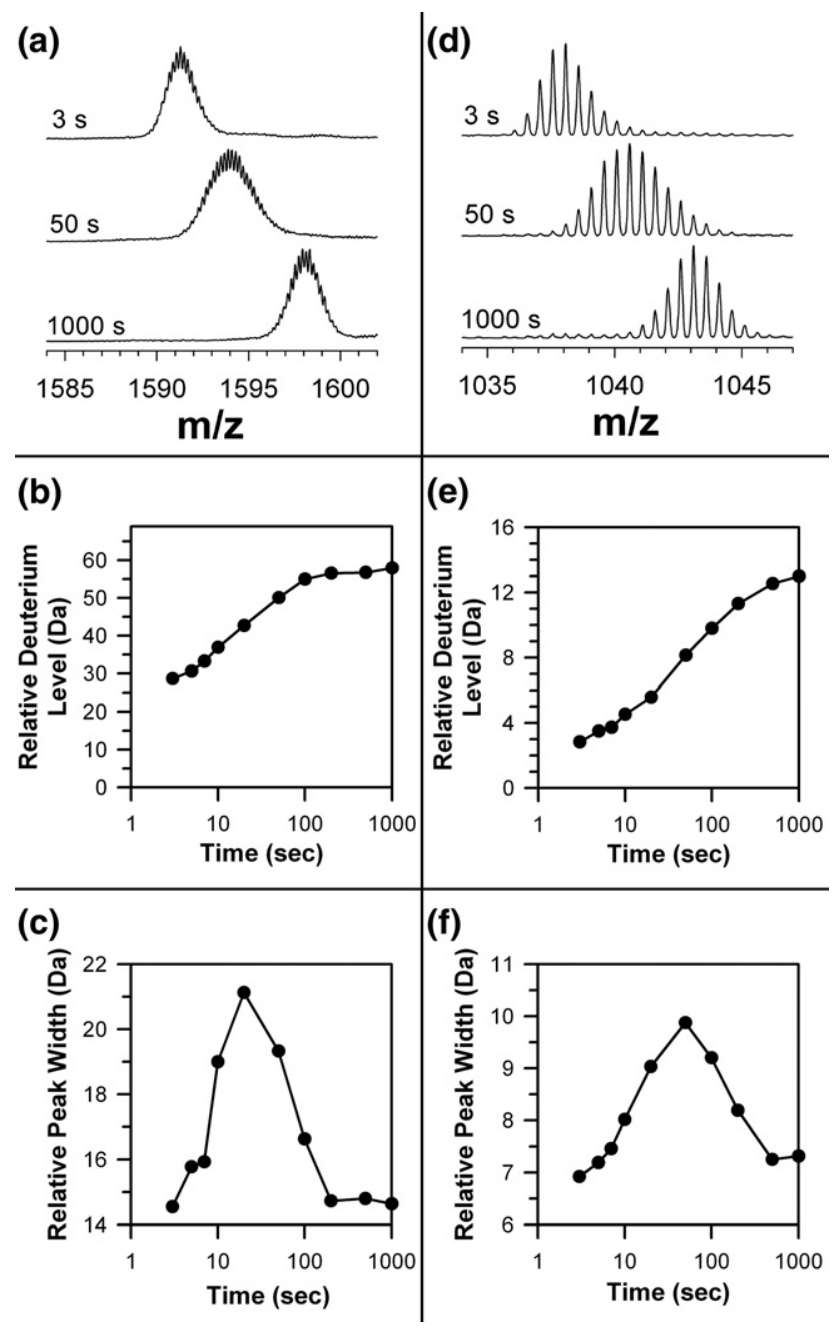

(f)

Figure 5. Width analysis of spectra displaying mixed, or less obvious EX1 kinetics. (a-c) Data for the Lck SH3 protein [19] where (a) raw spectra for selected time points, (b) deuterium uptake curve, and (c) peak-width analysis. (d-f) Data for the Lck SH3 peptide 44-61 where (d) raw spectra for selected time points, (e) deuterium uptake curve, and (f) peak-width analysis. All spectra for the entire time course for both protein and peptide are available in the Supplementary Material Figures 14s, 15s.

Changes to protein dynamics as a result of binding become very clear, as illustrated previously [19].

One desire would be a way to automate the analysis of HXMS data to automatically distinguish between EX2 and EX1 kinetic signatures. The DEX software package has been upgraded to include this capability. The software was modified from its first form [18] to process multiply charged species observed in electrospray data and a new algorithm was implemented to prepare peak-width plots. DEX removes the natural isotopic abundance from HXMS data and provides a readout of the populations of deuterons present at each exchange time point. The advantage of removing the stable natural isotopic abundance profile is to remove one element from the widening of the observed mass spectrum profile, thereby focusing the results on the real changes occurring during the experiment. The 
(a)

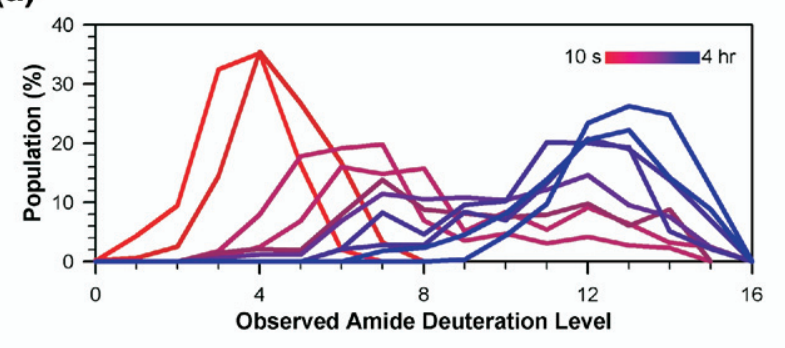

(b)

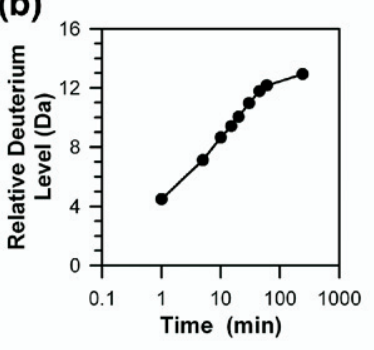

(c)

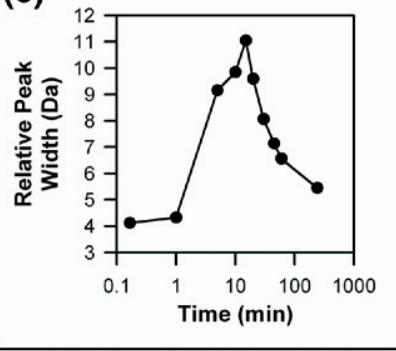

(d)
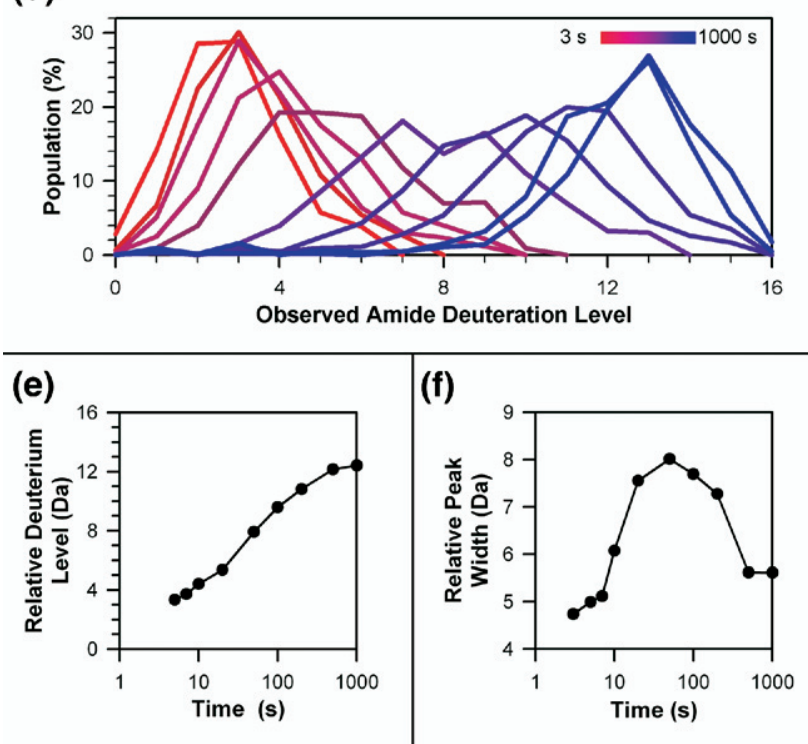

Figure 6. Analysis of EX1 kinetics with the DEX software package. The natural isotopic abundance was deconvoluted from the mass spectra of the Lyn SH3 99-116 peptide (a-c) [16] and the Lck SH3 peptide 44-61 (d-f) [19] with DEX [18]. (a) and (d) show the population of different observed deuteration levels for the full time courses. Coloring from red to blue indicates the progression of the deuterium exchange time as indicated in the legend. (b) and (e) show the centroids of the populations as a function of deuterium exchange time, and compare closely with the same uptake levels found for the same data without deconvolution (Figures $4 \mathrm{e}$ and 5e). (c) and (f) show the widths of the distributions at $20 \%$ of maximum, which are similar in shape, but not in magnitude due to the removal of the natural isotopic distribution (Figures $4 \mathrm{f}$ and 5f). All spectra for both time courses are available in the Supplementary Material Figures 13s and 15s.

same data presented in Figures $4 d-f$ and $5 d-f$ were processed with DEX and the results are shown in Figure 6. As shown in Figure 6a and d, the population distribution graphs indicate a significant widening at inter- mediate exchange times, similar to what was observed in raw spectra (Figures 4d, 5d, and Supplementary Materials section). DEX reliably reproduced the deuterium uptake curves (Figure 6b, e) and produced similar peak-width graphs (Figure 6c, f) as were previously obtained with manual processing (Figures 4f, 5f). The axes of the graphs are different because DEX removes the natural isotopic abundance from the raw data, but the relative change in magnitude on the $y$-axis and the time occurrence of the apex are effectively the same as the manually processed data. Overall, DEX automatically provides the same results in terms of peak-width analysis for an obvious EX1 case (Figure 6a-c) and for a less obvious case (Figure 6d-f). Simply by preparing peak-width curves, therefore, DEX can distinguish between data that has EX2 kinetics and data that has EX1 kinetics, principally by monitoring the change in slope (first derivative) in the peak-width plot.

Using DEX software, all information from HXMS that is desired can be obtained with relatively little effort. A manual interpretation of the raw spectra is still required and, in our opinion, necessary. Once the data has been found and fed into the DEX program, algorithms can be trusted to provide reliably accurate calculations. Human intervention is still necessary to observe the peaks, determine which peaks to process, eliminate overlaps by choosing scans in the chromatogram that reduce interference from nearby eluting peptides, etc. Or, if one chooses, all the peaks may be fed into DEX to deconvolute and analyze, and those that are deemed of acceptable quality can be later chosen using the DEX package's visual and numerical analysis tools. Once provided with good spectra, DEX can calculate, automatically and quickly, the deuterium incorporation in terms of explicit populations (Figure 6a and $\mathrm{d}$ ), provide the deuterium uptake plots (Figure $6 \mathrm{~b}$ and e), and finally make the peak width plots (Figure $6 \mathrm{c}$ and $\mathrm{f}$ ) to determine if EX1 kinetics are present. Automated generation of $t_{1 / 2}$ and relative involvement values for the EX1 event are under development.

\section{Conclusions}

As we have shown here, EX1 kinetics whether obvious or not, can be easily revealed by preparing a plot of the change in peak width with time. Realistic models of EX2 exchange show that there is little increase in peak width and that the worst case widening can actually be calculated for any sequence based on a simple model. These models are supported by empirical evidence of EX2 exchanging proteins when analyzed by the same peak width analysis technique. From plots of peak width, the half-life for EX1 events can be easily determined and the relative number of residues involved can be estimated. Although deconvolution of the natural isotope abundance from the hydrogen exchange mass spectrometry data results in narrower peak width overall, no new information is gained and the same ultimate conclusions are reached in terms of the presence of EX1, 
the half-life, and the residue involvement. It is therefore not necessary to prepare isotope depleted proteins in an effort to remove the natural isotope abundance from the width of peptide or protein distributions. The analysis of peak width provides a rapid, simple diagnostic method for identifying EX1 kinetic events that are revealed by hydrogen exchange mass spectrometry data and the process of distinguishing them from EX2 exchanging regions can be partially automated.

\section{Acknowledgments}

The authors thank Y. Wu and J. Hochrein for providing some of the raw mass spectrometry data. Funding for this project was provided by the NIH through grants R01-GM070590 (JRE) and R01-GM070996 (LFTE).

\section{Appendix}

The peak-width determination for peptides consisted of the following steps:

1. The peptide spectrum was smoothed twice with a four point Savitsky-Golay smoothing function using MassLynx (Waters). The smoothed spectrum was transferred into Excel as two columns of data: $\mathrm{m} / \mathrm{z}$ values and intensities, $I$.

2. The maximum peak in the isotopic envelope was identified.

3. Based on the charge state of the peptide, isotopic peaks in the envelope were identified in the spectrum. Starting at the maximum point, the spectrum was scanned in both directions to create a list of the isotopic peaks. The $i$ th point in the spectrum was designated as an isotopic peak if it satisfied the following criteria: (a) The intensity exceeded a userdefined threshold (typically 5\% of maximum spectral intensity). This threshold served to suppress noise in the spectrum. (b) The intensity represented a local maximum such that $I_{i}>I_{i \pm 1}>I_{i \pm 2}$. (c) The $\mathrm{m} / \mathrm{z}$ distance between the prospective peak and the previous peak (or the peak maximum) was equal to $1 / z$ within a user-specified tolerance (typically $\pm 0.03 \mathrm{Da}$ ).

4. The list of peaks was searched in ascending $\mathrm{m} / \mathrm{z}$ order to identify the two peaks that straddled 20\% of peak maximum. The $\mathrm{m} / \mathrm{z}$ value at an envelope intensity of $20 \%$ of peak maximum was determined using interpolation between these two peaks.

5. Step 4 was repeated with a search in descending $\mathrm{m} / \mathrm{z}$ order.

6. The relative peak width was determined from $z \times$ $\Delta m / z$.

Some peptide spectra did not have any peaks with intensities less than $20 \%$ of the peak maximum or peaks that straddled the $20 \%$ threshold existed only on one side of the maximum (typically, the high $\mathrm{m} / \mathrm{z}$ side). This is typical for +1 charge state peptides, especially when only slightly deuterated. Since the monoisotopic peak is the maximum peak, there are no peaks on the low $\mathrm{m} / \mathrm{z}$ side of the peak maximum. In these cases, the interpolation scheme automatically switched over from measuring the width of the peak-to-peak envelope to a direct measurement of the width of the isotopic envelope. For example, for an undeuterated +1 peptide, the low $\mathrm{m} / \mathrm{z}$ limit for the width measurement was set at the point at which the spectral intensity crossed $20 \%$ of the peak maximum (determined by interpolation) while the high $m / z$ limit was determined as described previously.

\section{References}

1. Miranker, A.; Robinson, C. V.; Radford, S. E.; Aplin, R. T.; Dobson, C. M. Detection of transient protein folding populations by mass spectrometry. Science 1993, 262, 896-900.

2. Engen, J. R.; Smith, D. L. Investigating the higher order structure of proteins: Hydrogen exchange, proteolytic fragmentation and mass spectrometry. Methods Mol. Biol. 2000, 146, 95-112.

3. Arrington, C. B.; Teesch, L. M.; Robertson, A. D. Defining protein ensembles with native-state $\mathrm{NH}$ exchange: Kinetics of interconversion and cooperative units from combined NMR and MS analysis. J. Mol. Biol. 1999, 285, 1265-1275.

4. Englander, S. W.; Kallenbach, N. R. Hydrogen exchange and structural dynamics of proteins and nucleic acids. Q. Rev. Biophys. 1984, 16, 521-655.

5. Englander, S. W. Protein folding intermediates and pathways studied by hydrogen exchange. Annu. Rev. Biophys. Biomol. Struct. 2000, 29, 213-238.

6. Sivaraman, T.; Robertson, A. D. Kinetics of conformational fluctuations by EX1 hydrogen exchange in native proteins. Methods Mol. Biol. 2001, $168,193-214$.

7. Hoofnagle, A. N.; Resing, K. A.; Ahn, N. G. Protein analysis by hydrogen exchange mass spectrometry. Annu. Rev. Biophys. Biomol. Struct. 2003, 32, 1-25.

8. Wales, T. E.; Engen, J. R. Hydrogen exchange mass spectrometry for the analysis of protein dynamics. Mass Spectrom. Rev. 2006, 25, 158-170.

9. Kaltashov, I. A.; Eyles, S. J. Studies of biomolecular conformations and conformational dynamics by mass spectrometry. Mass Spectrom. Rev. 2002, 21, 37-71.

10. Arrington, C. B.; Robertson, A. D. Kinetics and thermodynamics of conformational equilibria in native proteins by hydrogen exchange. Methods Enzymol. 2000, 323, 104-124.

11. Ferraro, D. M.; Lazo, N. D.; Robertson, A. D. EX1 hydrogen exchange and protein folding. Biochemistry 2004, 43, 587-594.

12. Roder, H.; Wagner, G.; Wuthrich, K. Amide proton exchange in proteins by EX1 kinetics: Studies of the basic pancreatic trypsin inhibitor at variable pH 2 and temperature. Biochemistry 1985, 24, 7396-7407.

13. Houliston, R. S.; Liu, C.; Singh, L. M.; Meiering, E. M. pH and urea dependence of amide hydrogen-deuterium exchange rates in the $\beta$ trefoil protein hisactophilin. Biochemistry 2002, 41, 1182-1194.

14. Maier, C. S.; Schimerlik, M. I.; Deinzer, M. L. Thermal denaturation of Escherichia coli thioredoxin studied by hydrogen/deuterium exchange and electrospray ionization mass spectrometry: Monitoring a 2-state protein unfolding transition. Biochemistry 1999, 38, 1136-1143.

15. Arrington, C. B.; Robertson, A. D. Microsecond to minute dynamics revealed by EX1-type hydrogen exchange at nearly every backbone hydrogen bond in a native protein. J. Mol. Biol. 2000, 296, 1307-1317.

16. Wales, T. E.; Engen, J. R. Partial unfolding of diverse SH3 domains on a wide time scale. J. Mol. Biol. 2006, 357, 1592-1604.

17. Xiao, H.; Hoerner, J. K.; Eyles, S. J.; Dobo, A.; Voigtman, E.; Mel'cuk, A. I.; Kaltashov, I. A. Mapping protein energy landscapes with amide hydrogen exchange and mass spectrometry: I. A generalized model for a 2-state protein and comparison with experiment. Protein Sci. 2005, 14, 543-557.

18. Hotchko, M.; Anand, G. S.; Komives, E. A.; Ten Eyck, L. F. Automated extraction of backbone deuteration levels from amide $\mathrm{H} /(2) \mathrm{H}$ mass spectrometry experiments. Protein Sci. 2006, 15, 583-601.

19. Weis, D. D.; Kjellen, P.; Sefton, B. M.; Engen, J. R. Altered dynamics in Lck SH3 upon binding to the LBD1 domain of Herpesvirus saimiri tip; unpublished.

20. Wu, Y.; Kaveti, S.; Engen, J. R. Extensive deuterium back-exchange in certain immobilized pepsin columns used for H/D exchange mass spectrometry. Anal. Chem. 2006, 78, 1719-1723.

21. Zhang, Z.; Smith, D. L. Determination of amide hydrogen exchange by mass spectrometry: A new tool for protein structure elucidation. Protein Sci. 1993, 2, 522-531.

22. Carter, J. M.; Gurevich, V. V.; Prossnitz, E. R.; Engen, J. R. Conformational differences between arrestin2 and preactivated mutants as revealed by hydrogen exchange mass spectrometry. J. Mol. Biol. 2005, 351, 865-878. 
23. Fu, C. Y.; Prevelige, P. E. Jr. Dynamic motions of free and bound O29 scaffolding protein identified by hydrogen deuterium exchange mass spectrometry. Protein Sci 2006; in press

24. Hochrein, J. M.; Lerner, E. C. Schiavone, A. P.; Smithgall, T. E.; Engen, J. R. An examination of dynamics crosstalk between $\mathrm{SH}_{2}$ and $\mathrm{SH} 3$ domains by hydrogen/deuterium exchange and mass spectrometry. Protein Sci. 2006, 15, 65-73.

25. Gmeiner, W. H.; Xu, I.; Horita, D. A.; Smithgall, T. E.; Engen, J. R.; Smith, D. L.; Byrd, R. A. Intramolecular binding of a proximal PPII helix to an SH3 domain in the fusion protein SH3Hck:PPIThGAP. Cell. Biochem. Biophys. 2001, 35, 115-126.

26. Chung, E. W.; Nettleton, E. J.; Morgan, C. J.; Gross, M.; Miranker, A.; Radford, S. E.; Dobson, C. M.; Robinson, C. V. Hydrogen exchange properties of proteins in native and denatured states monitored by mass spectrometry and NMR. Protein Sci. 1997, 6, 1316-1324.
27. Molday, R. S.; Englander, S. W.; Kallen, R. G. Primary structure effects on peptide group hydrogen exchange. Biochemistry 1972, 11, 150-158.

28. Bai, Y.; Milne, J. S.; Mayne, L.; Englander, S. W. Primary structure effects on peptide group hydrogen exchange. Proteins: Struct. Funct. Genet. 1993, 17, 75-86.

29. Connelly, G. P.; Bai, Y.; Jeng, M.-F.; Englander, S. W. Isotope effects in peptide group hydrogen exchange. Proteins: Struct. Funct. Genet. 1993, 17, 87-92.

30. Engen, J. R.; Smithgall, T. E.; Gmeiner, W. H.; Smith, D. L. Identification and localization of slow, natural, cooperative unfolding in the hematopoietic cell kinase $\mathrm{SH} 3$ domain by amide hydrogen exchange and mass spectrometry. Biochemistry 1997, 36, 14384-14391.

31. Smith, D. L.; Deng, Y.; Zhang, Z. Probing the noncovalent structure of proteins by amide hydrogen exchange and mass spectrometry. J. Mass. Spectrom. 1997, 32, 135-146. 